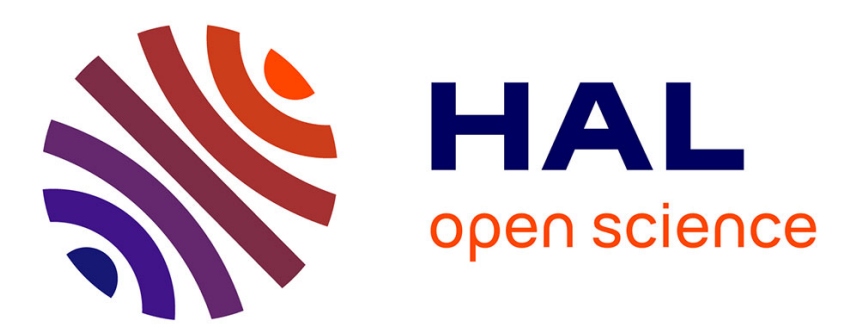

\title{
Functional, Molecular and Proteomic Characterization of Bone Marrow Mesenchymal Stem Cells in Rheumatoid Arthritis
}

M. Kastrinaki, P. Sidiropoulos, S. Roche, J. Ringe, Sylvain Lehmann, H. Kritikos, V. Vlahava, B. Delorme, G. Eliopoulos, Christian Jorgensen, et al.

\section{To cite this version:}

M. Kastrinaki, P. Sidiropoulos, S. Roche, J. Ringe, Sylvain Lehmann, et al.. Functional, Molecular and Proteomic Characterization of Bone Marrow Mesenchymal Stem Cells in Rheumatoid Arthritis. Annals of the Rheumatic Diseases, 2008, 67 (6), pp.741-749. 10.1136/ard.2007.076174 . hal-00287919

\section{HAL Id: hal-00287919 https://hal.science/hal-00287919}

Submitted on 21 Dec 2017

HAL is a multi-disciplinary open access archive for the deposit and dissemination of scientific research documents, whether they are published or not. The documents may come from teaching and research institutions in France or abroad, or from public or private research centers.
L'archive ouverte pluridisciplinaire HAL, est destinée au dépôt et à la diffusion de documents scientifiques de niveau recherche, publiés ou non, émanant des établissements d'enseignement et de recherche français ou étrangers, des laboratoires publics ou privés. 


\title{
Functional, molecular and proteomic characterisation of bone marrow mesenchymal stem cells in rheumatoid arthritis
}

\author{
M-C Kastrinaki, ${ }^{1}$ P Sidiropoulos, ${ }^{2}$ S Roche, ${ }^{3}$ J Ringe, ${ }^{4}$ S Lehmann, ${ }^{3}$ H Kritikos, ${ }^{2}$ \\ V-M Vlahava, ${ }^{1}$ B Delorme, ${ }^{5}$ G D Eliopoulos, ${ }^{1}$ C Jorgensen, ${ }^{6}$ P Charbord, ${ }^{5}$ T Häupl, ${ }^{4}$ \\ D T Boumpas, ${ }^{2}$ H A Papadaki ${ }^{1}$
}

${ }^{1}$ Department of Haematology, University of Crete School of Medicine, Heraklion, Crete, Greece; ${ }^{2}$ Department of Rheumatology, Clinical Immunology and Allergiology, University of Crete School of Medicine, Heraklion, Crete, Greece; ${ }^{3}$ Institut de Génétique Humaine, UPR1142 CNRS,

Montpellier, France;

${ }^{4}$ Department of Rheumatology and Clinical Immunology, Charité Universitätsmedizin, Berlin, Germany; ${ }^{5}$ Equipe INSERMESPRI/EA-3855, Faculty of

Medicine, University Francois

Rabelais, Tours, France;

${ }^{6}$ Service d'Immuno-

Rhumatologie, Hopital

Lapeyronie, Montpellier, France

Correspondence to:

H A Papadaki, University of Crete School of Medicine, P.O. Box 1352, Heraklion, Crete, Greece; epapadak@med.uoc.gr

Accepted 17 September 2007 Published Online First

5 October 2007

\section{ABSTRACT}

Objective: Bone marrow (BM) mesenchymal stem cells (MSCs) are being considered as potential therapeutic agents in various inflammatory autoimmune diseases for their tissue-repair and anti-inflammatory tissue-protective properties. This study investigates the reserves and function, the molecular and proteomic profile and the differentiation potential of BM MSCs in patients with active rheumatoid arthritis (RA).

Methods: We evaluated the frequency of MSCs in the BM mononuclear cell fraction using a limiting dilution assay, the proliferative/clonogenic potential and the capacity of cells to differentiate towards the osteogenic/ chondrogenic/adipogenic lineages using appropriate culture conditions. We also assessed the molecular and proteomic characteristics in terms of inflammatory cytokine gene and protein expression, the relative telomere length and the survival characteristics of BM MSCs.

Results: MSCs from patients with RA ( $n=26)$ and ageand sex-matched healthy individuals $(n=21)$ were similar in frequency, differentiation potential, survival, immunophenotypic characteristics, and protein profile. Patient MSCs, however, had impaired clonogenic and proliferative potential in association with premature telomere length loss. Transcriptome analysis revealed differential expression of genes related to cell adhesion processes and cell cycle progression beyond the $\mathrm{G} 1$ phase. Previous treatment with methotrexate, corticosteroids, anti-cytokine and biological agents or other disease-modifying antiinflammatory drugs did not correlate with the clonogenic and proliferative impairment of BM MSCs.

Conclusion: In spite of some restrictions related to the impaired clonogenic and proliferative potential, our findings support the use of autologous BM MSCs in RA and may have important implications for the ongoing efforts to repair tissue injury commonly seen in the course of the disease.

Adult multipotential stem cells isolated from a variety of tissues have been demonstrated to participate in tissue homeostasis and repair under the influence of appropriate signals. Among the most well characterised types of adult stem cells are the bone marrow (BM) derived mesenchymal stem cells (MSCs). ${ }^{12}$ These cells reside in BM stroma providing the supporting feeder cells necessary for the haematopoietic progenitor cell growth but they may also differentiate into cells of connective tissue such as osteocytes, chondrocytes, tenocytes, adipocytes and smooth muscle cells. ${ }^{34}$
There is currently great interest in exploring the potential use of MSCs in regenerative medicine with particular target the bone and cartilage defects. ${ }^{5-10}$

Rheumatoid arthritis (RA) is a systemic autoimmune disease characterised by cartilage and bone destruction associated with local production of inflammatory mediators such as tumour necrosis factor $\alpha(\mathrm{TNF} \alpha)$ and interleukin (IL)1 $\beta$. Joint destruction renders RA a candidate disease for cartilage and bone repair using MSCs. There are, however, some specific issues concerning the reserves and function of BM MSCs in RA that need to be addressed before proceeding to clinical application. In addition to the concept that BM MSCs may be primarily involved in joint damage in $\mathrm{RA},{ }^{11-13}$ there are studies demonstrating that increased local production of $\mathrm{TNF} \alpha$ may injure the BM microenvironment and may affect the reserves of BM haematopoietic progenitor cells. ${ }^{14}$ Accordingly, a critical issue is whether BM MSCs are also depleted or functionally altered in RA.

This study evaluates the reserves and function of BM MSCs in patients with RA in terms of their proliferative, clonogenic and differentiation potential. Molecular and proteomic characteristics of patient MSCs with special focus on inflammatory cytokine gene and protein expression were also studied.

\section{PATIENTS AND METHODS}

\section{Patients}

We studied 26 patients with active RA ${ }^{15}{ }^{16}$ and 21 healthy individuals, age- and sex-matched with the patients (table 1). The Institutional ethics committee approved the study and informed consent according to the Helsinki Declaration was obtained from all subjects.

\section{MSC culture and differentiation}

BM mononuclear cells (BMMCs) obtained from posterior iliac crest aspirates were cultured in Dulbecco modified Eagle medium, low glucose (DMEM-LG; Gibco/Invitrogen, Paisley, Scotland)/ $10 \%$ foetal calf serum (FCS; Hyclone, Logan, Utah, USA)/100 IU/ml penicillin-streptomycin (MSC medium) and MSCs were grown as previously described. ${ }^{17}{ }^{18}$ Cell-free supernatants were stored ($72^{\circ} \mathrm{C}$ ) for cytokine measurements by ELISA (Quantikine; R\&D Systems, Minneapolis, 


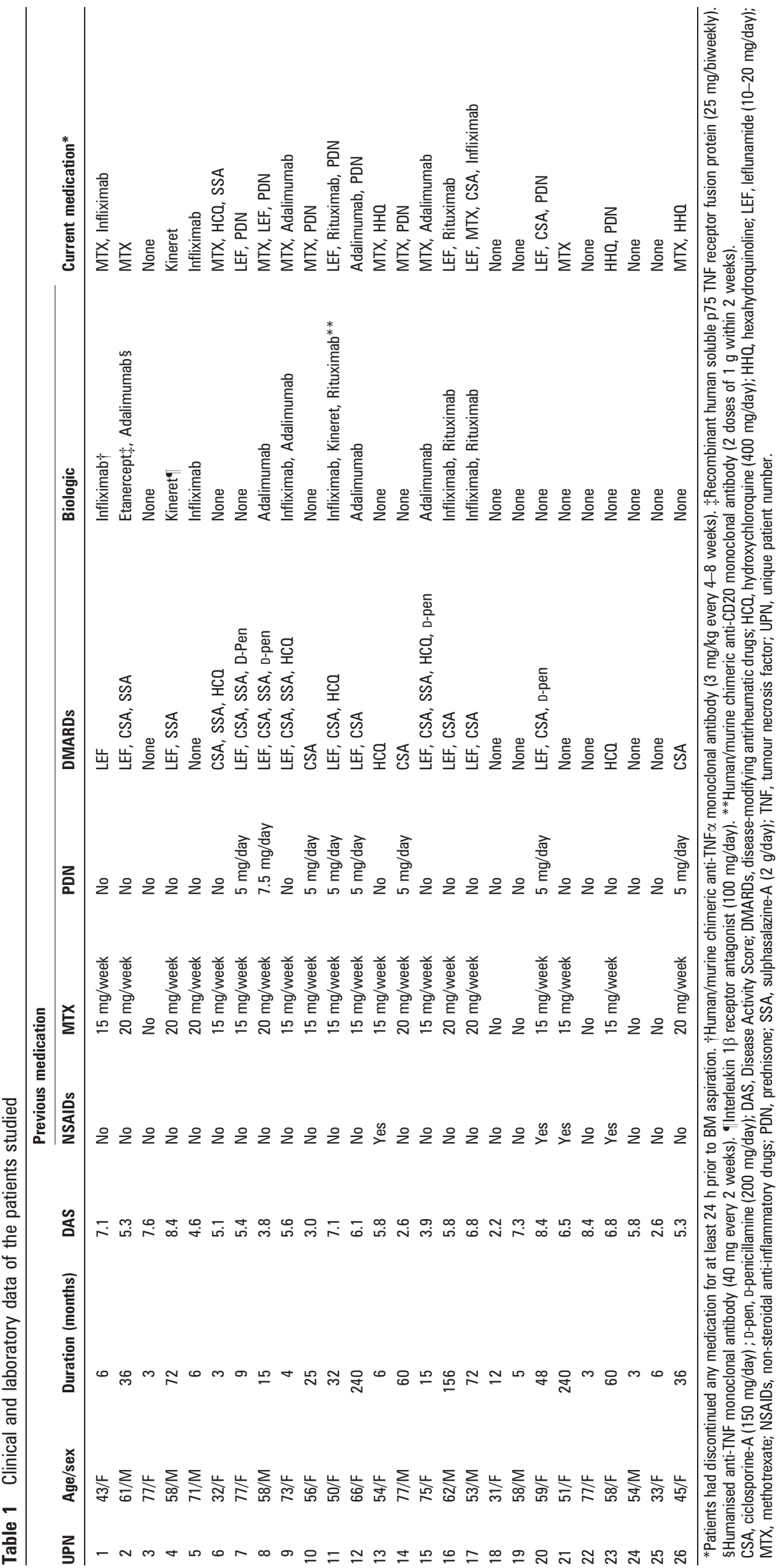



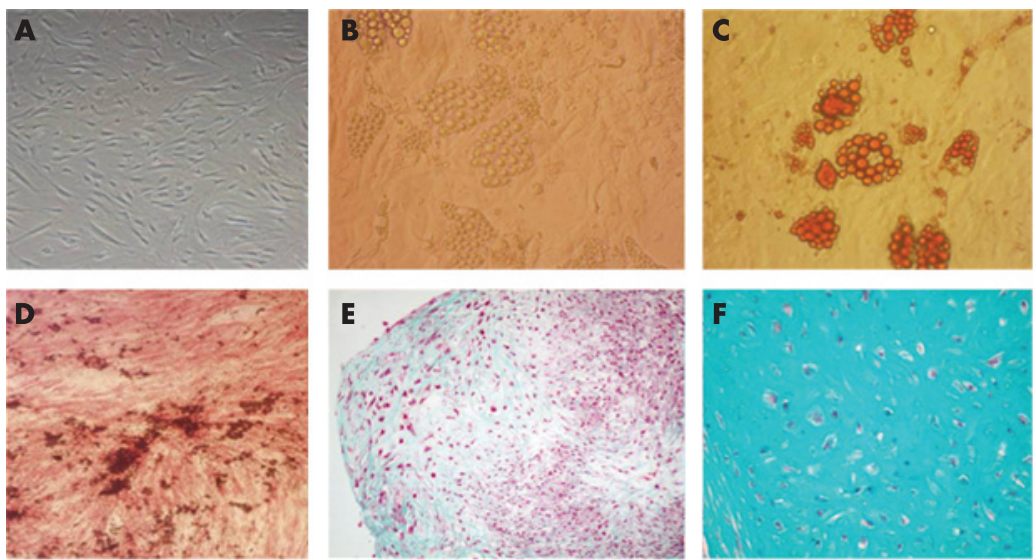

G
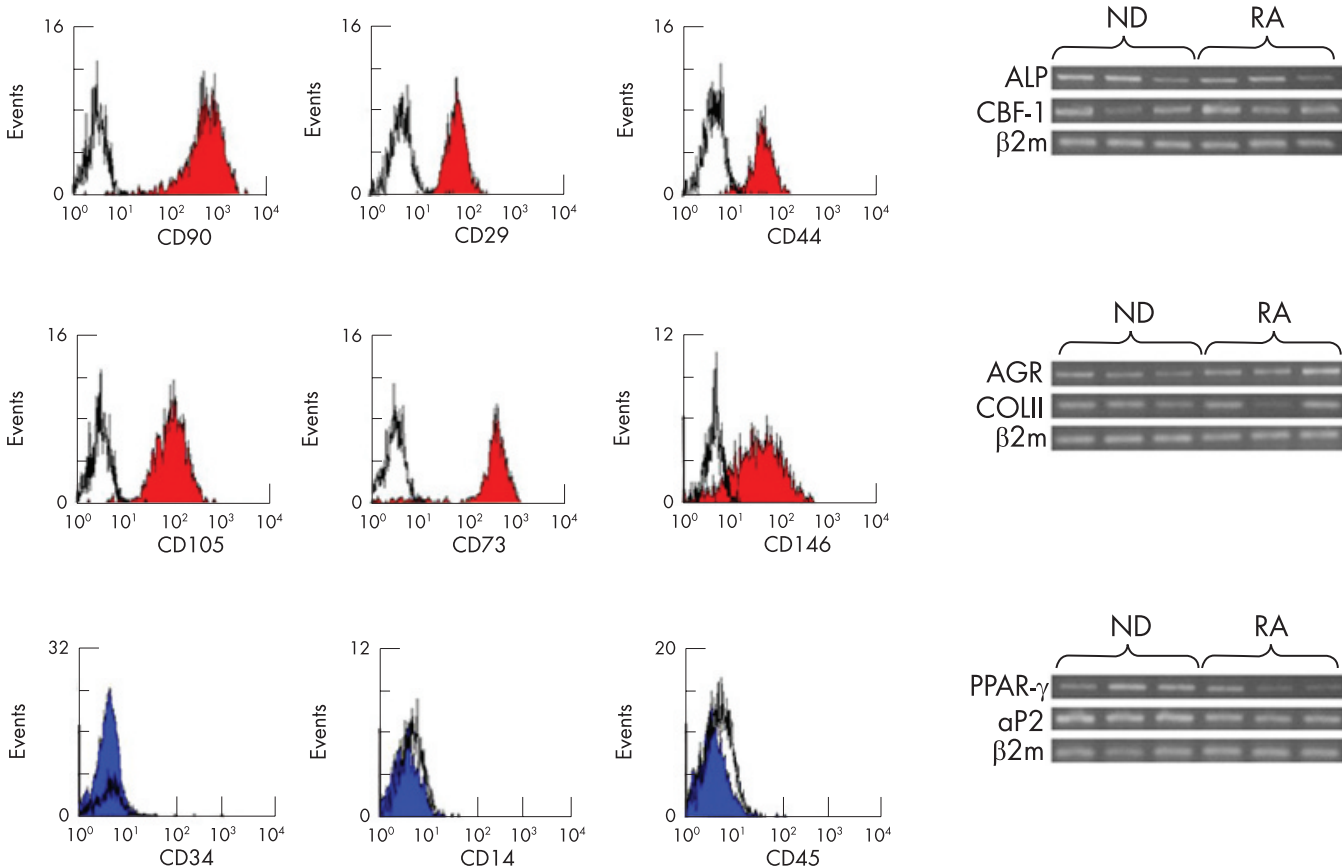
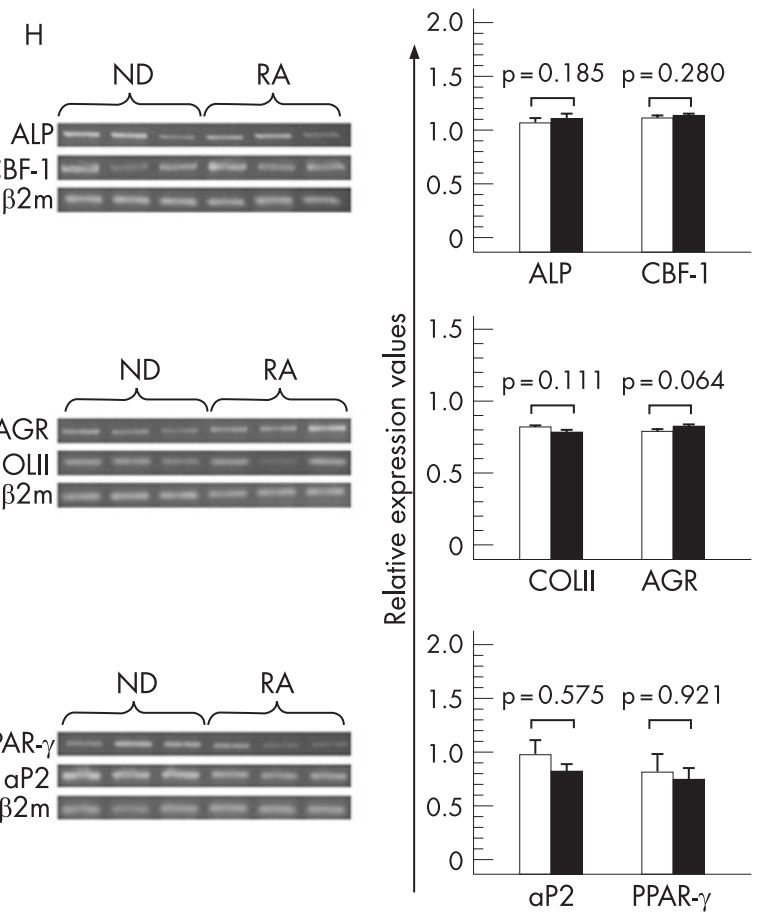

Figure 1 Differentiation potential and immunophenotypic characteristics of mesenchymal stem cells (MSCs). The upper panel shows undifferentiated MSCs from passage 2 (P2) (A) and differentiated cells towards the adipogenic (B and C), osteogenic (D) and chondrogenic (E and F) lineages from a representative patient with rheumatoid arthritis (RA). Cell differentiation was identified with Oil Red 0 (C), alkaline phospahatse (ALP)/Non Kossa (D), Masson (E) and Alcian blue (F) staining. G. Immunophenotypic characteristics of patient MSCs at P2. The red and blue histograms depict the positive and negative markers, respectively, compared to the isotype-matched negative controls (open histograms). H. Specific gene mRNA expression (on the left) of P2 BM MSCs upon differentiation towards the osteogenic, chondrogenic and adipogenic lineages in representative $R A(n=3)$ and control $(n=3)$ subjects as well as cumulative data (mean relative values $(S E M))$ of specific gene mRNA expression from all RA (solid squares) and controls (open squares) studied (on the right). Comparison has been performed by means of the non-parametric Mann-Whitney U test.

Minnesota, USA). Trypsinised MSCs from passage (P)-2 were induced for differentiation (fig $1 \mathrm{~A}-\mathrm{F}$ ).

MSC adipogenic and osteogenic differentiation was induced as previously described and assessed by Oil Red $\mathrm{O}$ and alkaline phosphatase (ALP)/Von Kossa stains, respectively. ${ }^{19}$ For chondrogenic induction, MSCs were pelleted and cultured in DMEM high glucose (DMDM-HG, Gibco), supplemented with $6.25 \mu \mathrm{g} /$ $\mathrm{ml}$ insulin, $6.25 \mu \mathrm{g} / \mathrm{ml}$ transferrin, $1.33 \mu \mathrm{g} / \mathrm{ml}$ linoleic acid, $1.25 \mathrm{mg} / \mathrm{ml}$ bovine serum albumin, $1 \mathrm{mM}$ sodium pyruvate, $0.17 \mathrm{mM}$ ascorbate-2-phosphate, $0.1 \mu \mathrm{M}$ dexamethasone, $0.35 \mathrm{mM}$ L-proline, $6.25 \mathrm{ng} / \mathrm{ml}$ selenous acid and $0.01 \mu \mathrm{g} / \mathrm{ml}$ transforming growth factor (TGF) $-\beta_{3} \quad\left(\mathrm{R} \& \mathrm{D}\right.$ Systems). ${ }^{20}$ Chondrogenic differentiation was identified with Alcian blue and Masson trichrome stains. Reagents were purchased from Sigma (St Louis, Minnesota, USA) unless otherwise indicated.

\section{MSC immunophenotypic and survival characteristics}

Trypsinised MSCs from passages 1-6 (P1-P6) were immunophenotypically characterised by flow-cytometry, using antiCD29 (4B4; Cyto-Stat, Beckman-Coulter, Fullerton, California, USA), anti-CD44 (J173; Immunotech/Coulter, Marseille, France), anti-CD73 (AD2; Pharmingen, San Diego, California, USA), anti-CD90 (F15.42; Immunotech/Coulter), anti-CD105 (SN6; Caltag, Burlingame, California, USA), anti-CD146 (P1H12; Pharmingen), anti-CD45 (IMMU19.2; Immunotech/ Coulter), anti-CD14 (RMO52; Immunotech/Coulter) and antiCD34 (OBend10; Beckman-Coulter) monoclonal antibodies.

Apoptosis was studied by flow-cytometry using 7-aminoactinomycin-D (7-AAD; Calbiochem-Novabiochem, La Jolla, California, USA). ${ }^{14}{ }^{21}$ Results were expressed as 7-AAD ${ }^{\text {neg }}$ (live), 7-AAD ${ }^{\text {dim }}$ (early apoptotic) and 7-AAD bright (late apoptotic) cells (fig 2A,B). 
Figure 2 Survival characteristics of mesenchymal stem cells (MSCs). MSCs from passages 1-6 (P1-P6) were stained with 7-aminoactinomycin-D (7-AAD) and analysed by flow cytometry for the study of apoptosis. Scattergram B shows the proportion of 7-AAD neg (R2; live), 7$A A D^{\operatorname{dim}}$ (R3; early apoptotic) and 7$A A D^{\text {bright }}$ ( $R 4$; late apoptotic) cells in the gate of $R 1$ (scattergram $A$ ) representing MSCs from P2. Bars in the lower panel represent the mean (SEM) proportion of live, early and late apoptotic cells timecourse in patients (C) and healthy controls (D) analysed as above. No statistically significant differences were documented between patients and controls by means of two-way analysis of variance (ANOVA).

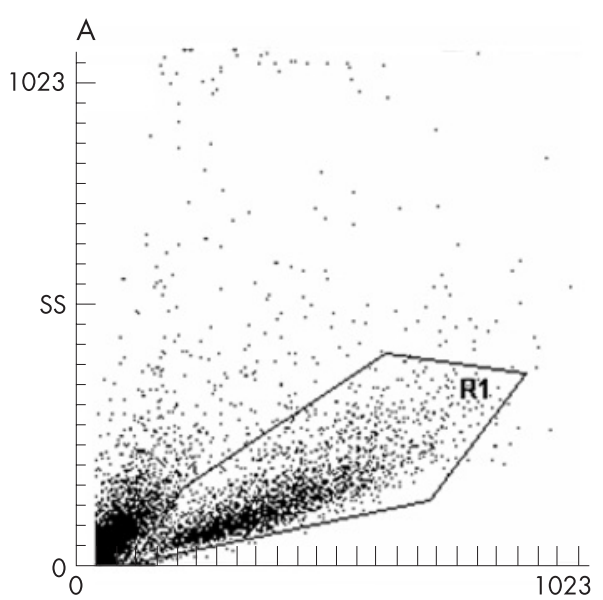

FS

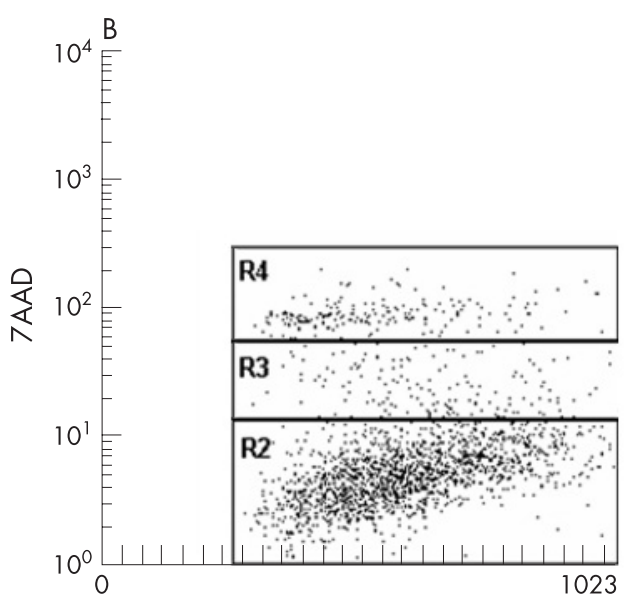

FS

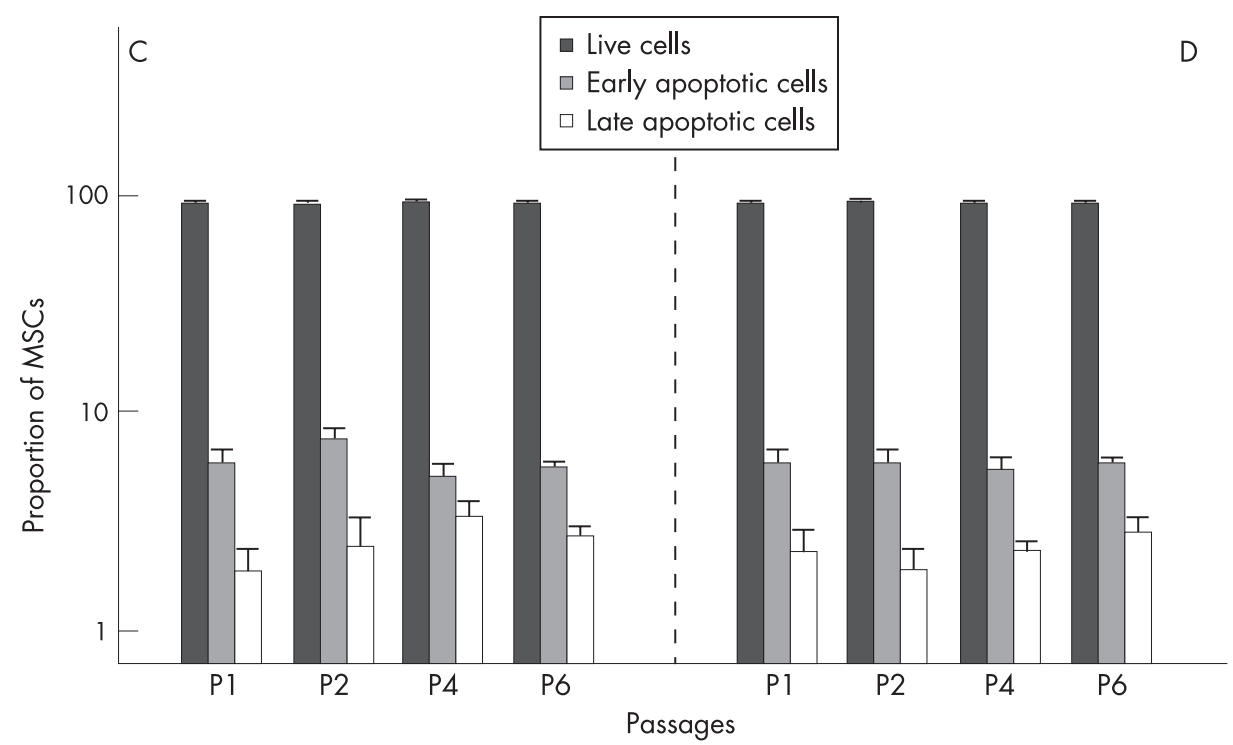

\section{MSC quantification in the BMMC fraction}

A limiting dilution assay (LDA) was used to evaluate MSC frequency within BMMCs following 6-week culture of seven different BMMC concentrations (250-10 000 cells/well) in 96well plates. ${ }^{17}$ Wells with $>50$ adherent spindle shaped cells were considered positive and MSC frequency corresponded to the dilution resulting in $37 \%$ negative wells. ${ }^{22}$

\section{Clonogenic potential and proliferative potential of MSCs}

A colony forming unit fibroblastic assay (CFU-F) was also used to evaluate MSC frequency within BMMCs. Briefly, day-0 BMMCs were seeded at three different concentrations, expanded for 14 days and CFU-F number was estimated using linear regression analysis. The clonogenic potential of trypsinised MSCs from P1-P6 was also evaluated by CFU-F. MSC proliferative potential was evaluated by a methyl triazolyl tetrazolium (MTT) based assay in P2 and also by estimating population doubling time through P1-P6.

\section{RT-PCR}

Total RNA isolated from P2 and differentiated MSCs (RNeasy mini kit; Oiagen $\mathrm{GmbH}$, Hilden, Germany) was reverse transcribed (SUPERSCRIPT II; Gibco) and amplified by RTPCR. Products were normalised according to $\beta_{2}$-microglobulin $\left(\beta_{2} \mathrm{~m}\right)$ using the ImageJ densitometry analysis system (http:// rsb.info.nih.gov/ij/). Primer sequences are listed in supplementary table 1 .

\section{Real time quantitative PCR}

\section{IL1 $\beta$ and TNF $\alpha$ expression}

Platinum ${ }^{\circledR} \mathrm{qPCR}$ SuperMix-UDG (Invitrogen) and TaqMan ${ }^{\circledR}$ Gene Expression Assays (Applied Biosystems; Foster City, California, USA) were used for IL1 $\beta$, TNF $\alpha$ and glyceraldehyde 3-phosphate dehydrogenase (GAPDH) identification. Measurements were performed using the ABI Prism 7000 System (Applied Biosystems). Results were normalised according to GAPDH quantity.

\section{Telomere length measurement}

DNA was extracted from P2 MSCs (Qiagen). $\beta$-Globin was used as control single copy gene. ${ }^{23}{ }^{24} \mathrm{iTaq}$ SYBR Green Supermix with ROX (Biorad, Hercules, California, USA) was used for the reactions. Primer sequences are listed in supplementary table 1. Telomere length was reflected by the relative telomere/singlecopy-gene ratio $(\mathrm{T} / \mathrm{S}): \mathrm{T} / \mathrm{S}=2^{-\Delta \mathrm{Ct}}\left(\Delta \mathrm{Ct}=\mathrm{Ct}^{\text {telomere }}-\mathrm{Ct}^{\beta-\text {-globin }}\right)$.

\section{Two-dimensional gel electrophoresis}

Whole cell lysates were obtained in $8 \mathrm{M}$ urea, $2 \mathrm{M}$ thiourea, $4 \%$ 3-[(3-cholamidopropyl)dimethylammonio]-1-propanesulfonate 
(CHAPS), $50 \mathrm{mM}$ dithiothreitol (DTT) (Sigma). For the first dimension, $30 \mu \mathrm{g}$ proteins from P2 MSCs were added to rehydration buffer. Immobilised $\mathrm{pH}$ gradient (IPG) strips $(\mathrm{pH}$ 3-10) were re-hydrated and focalised (70 $000 \mathrm{v} / \mathrm{h}$ ). For the second dimension, strips were equilibrated and proteins were separated by SDS-PAGE. Gels were stained with silver nitrate ${ }^{25}$ and scanned using the Labscan-3 software (GE Healthcare; Little Chalfont, Buckinghamshire, UK) after calibration by the Kaleidoscope LaserSoft Imaging software (Kodak ref: R020123; Eastman Kodak, Rochester, New York, USA). Spot detection and quantitation were performed by ImageMaster 2D Platinum software (GE Healthcare).

\section{Microarray experiments}

Hybridisation on HG-U133 Plus 2.0 microarrays was performed according to manufacturer (Affymetrix; Santa Clara, California, USA). Arrays were washed, stained and scanned on a Hewlett Packard Genearray Scanner (Affymetrix). Affymetrix GCOS 1.2 software was used to control washing and scanning, generate DAT/CEL/EXP files and process data. Group comparison and gene retrieval was performed by $\mathrm{SiPaGene}$ (BioRetis $\mathrm{GmbH}$; Berlin, Germany). Cluster analysis was calculated with Genesis (A. Sturn; Graz University, Graz, Austria).

\section{Statistical analysis}

Data were analysed by Mann-Whitney $U$ test, one-way and two-way analysis of variance (ANOVA) tests (GraphPad Software; San Diego, California, USA). Two-dimensional gel electrophoresis data were analysed by the Hierarchical Clustering Explorer 3.0 software (http://www.cs.umd.edu/ hcil/multi-cluster/) and Pearson correlation coefficient. ${ }^{26}$ Grouped data are expressed as mean (SD).

\section{RESULTS}

\section{MSC immunophenotype and differentiation potential}

Immunophenotypic analysis at the end of each passage demonstrated that cultures constituted of a homogenous cell population positive for CD73, CD90, CD146, CD105, CD29, CD44 and negative for CD45, CD14, CD34 surface antigens (fig 1G). ${ }^{19}$

Culture-expanded MSCs from patients were able to differentiate into osteogenic/chondrogenic/adipogenic lineages as shown by the respective cytochemical staining (fig 1B-F). Furthermore, the osteogenic/chondrogenic/adipogenic differentiating capacity assessed by the relative expression of ALP and CBF-1 mRNA, COLLII and AGR (aggrecan) mRNA, and aP2 (adipose fatty-acid binding protein 2) and peroxisome proliferator-activated receptor (PPAR)- $\gamma$ mRNA expression, respectively, did not differ between patients and controls, suggesting normal differentiation potential of RA MSCs (fig $1 \mathrm{H}$ ).

\section{MSC frequency in the BMMC fraction}

The estimated frequency of MSCs in the BMMC fraction did not differ significantly between patients (20.74 (12.41)/10 BMMCs) and controls (23.90 (15.65)/10 $\mathrm{BMMCs})$ $(p=0.8894)$ by LDA. Quantification of BM MSCs by CFU-F analysis at day 0 was in accordance with LDA data. CFU-F number obtained by day- 0 MSCs did not differ significantly between patients (18.42 (13.75)/10 5 BMMCs) and controls (19.85 (18.96)/105 BMMCs) ( $p=0.3464)$, further suggesting normal numbers of MSCs in RA. No statistically significant difference was documented between the LDA and CFU-F assays in the estimation of MSC frequency in either patients $(p=0.210)$ or healthy controls $(p=0.199)$.

\section{MSC clonogenic and proliferative potential}

The growth potential of BM MSCs in patients with RA was studied by evaluating their clonogenic and proliferative capacity over time. CFU-F recovery by culture-expanded MSCs was significantly lower in patients with RA compared to controls through $\mathrm{P} 1-\mathrm{P} 6(\mathrm{p}<0.01)$ (fig $3 \mathrm{~A})$. In association to the lower clonogenic potential, patient MSCs displayed also defective proliferative capacity compared to controls as estimated by the population doubling time over P1-P6 $(p<0.001)$ (fig 3B). These findings were further substantiated by the MTT assay; as shown in fig $3 \mathrm{C}$ depicting results from a representative passage (P2), the number of live cells remained significantly lower in patients with RA compared to controls $(\mathrm{p}<0.001)$.

The majority of patients had previously been treated with cytotoxic and/or immune suppressant or anti-cytokine agents. To exclude the possibility of a drug-related damage of MSCs, a subset analysis was performed in the group of previously treated $(n=20)$ and untreated $(n=6)$ patients. Compared to healthy controls, treated and untreated patient groups displayed lower CFU-F recovery through P1-P6 $(p<0.001$ and $p<0.05$, respectively). Similarly, treated and untreated patients displayed increased MSC doubling time $(p<0.001$ and $p<0.05$, respectively) and defective proliferative potential in the MTT assay $(p<0.001$ and $p<0.05$, respectively) compared to controls. However, no statistically significant differences were found between the two patient groups in the above parameters. Thus drug-induced damage is unlikely to be the cause of the defective clonogenic/proliferative potential of MSCs in RA.

\section{MSC survival characteristics}

The impaired clonogenic and proliferative ability of MSCs in patients with RA might have resulted from increased cell apoptosis. The total proportion of apoptotic cells ranged from $7.68(3.70) \%(\mathrm{P} 1)$ to $8.24(2.01) \%(\mathrm{P} 6)$ in the patients and from 8.25 (4.15)\% (P1) to 8.24 (2.85)\% (P6) in the controls. Overall, no statistically significant differences were found between patients and controls in the proportion of early $\left(\mathrm{F}=0.8206<\mathrm{F}^{1} 84\right.$, not significant) or late apoptotic $\left(\mathrm{F}=0.9977<\mathrm{F}^{1}{ }_{84}\right.$, not significant) cells throughout P1-P6 culture period, suggesting that the impaired clonogenic and proliferative potential of MSCs in RA is not due to accelerated cell apoptosis (fig 2C,D).

\section{Cytokine production by MSCs}

To investigate whether BM MSCs from patients with RA display abnormal cytokine production similar to RA synovial fibroblasts $^{27}$ we initially assessed IL1 $\beta$ and TNF $\alpha$ production. The relative IL1 $\beta / \mathrm{GAPDH}$ and TNF $\alpha / \mathrm{GAPDH}$ mRNA levels at P2 did not differ significantly between patients and controls (0.059 (0.029) and $0.040(0.022)$, respectively). Similar to the mRNA data, protein levels of IL1 $\beta$ and TNF $\alpha$ determined by ELISA in culture supernatants did not differ significantly between patients and controls through P2-P6 $\left(F=0.1474<\mathrm{F}^{1} 48\right.$ and $\mathrm{F}=1.2373<\mathrm{F}_{48}^{1}$, respectively).

The levels of the MSC growth-promoting cytokines vascular endothelial growth factor and stromal-derived factor- 1 and the values of the inhibitory/inflammatory cytokines IL6, TGF- $\beta_{1}$, IL8 and IL15 in culture supernatants through P2-P6 did not differ significantly between patients and controls. These data indicate that abnormal cytokine production by MSC per se seems unlikely to be the cause of the impaired growth potential of MSCs in RA. 
Figure 3 Clonogenic/proliferative potential and relative telomere length of mesenchymal stem cells (MSCs). The left panel depicts the mean (SEM) number from colony forming unit fibroblastic assay (CFU-F) (A) and days for population doubling (B) over passages 1-6 (P1-P6) as well as the mean (SEM) optical density corresponding to the number of live cells over 13-day culture using the methyl triazolyl tetrazolium (MTT) assay in P2 (C). The right panel illustrates the correlation (regression line within 95\% confidence limits) between age and relative MSC telomere/single-copy-gene values (diagrams $D$ and $E$, respectively) and the mean (SEM) relative telomere length per decade of years (F) in RA (solid squares) and normal (open squares) subjects. Analyses have been performed by two-way analysis of variance (ANOVA).
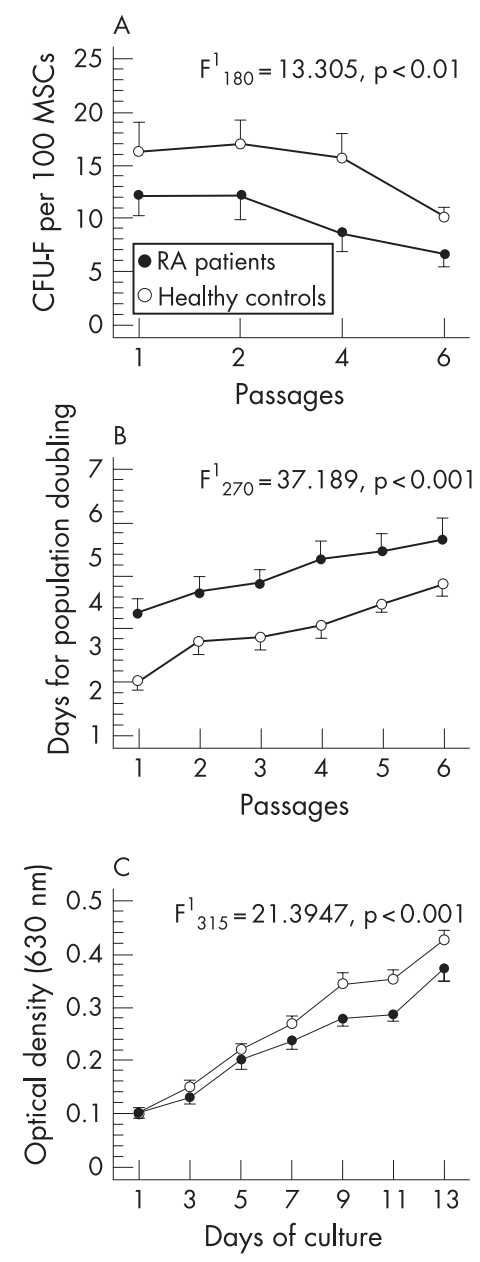
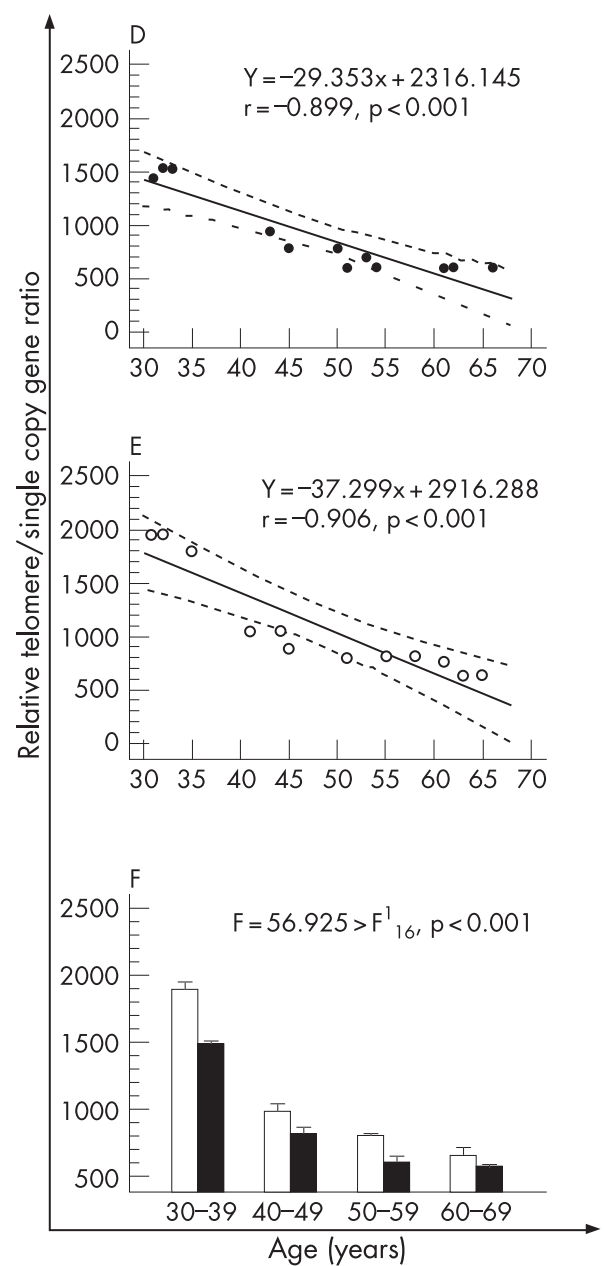

\section{Proteomic analysis}

Two-dimensional electrophoresis was performed using P2 MSCs in patients with RA $(n=10)$ and healthy controls $(n=6)$ (fig 4A,B). By using the Hierarchical Clustering method, we could not define any cluster that might discriminate patient and control cells (fig 4C). The Pearson correlation coefficient was not significantly different between patient and control cells $(r=0.933(0.022)$ and $r=0.929(0.020)$, respectively). These data corroborate the lack of significant changes in cytokine production between patients and controls.

\section{MSC telomere length}

Decreased cellular growth associated with premature telomeric loss has previously been described in periarticular osteoblasts in RA. ${ }^{28}$ We therefore evaluated the relative telomere length (RTL) of MSCs from P2 in 12 patients and 12 age- and sex-matched healthy controls. An age-related decrease of the RTL as estimated by the relative $\mathrm{T} / \mathrm{S}$ ratio was observed in patients $(r=-0,899, p<0.001)$ and controls $(r=-0.906, p<0.001)$ (fig 3D,E). To characterise patient MSC RTLs appropriate or inappropriate for a given age, we defined the observed/predicted $\mathrm{RTL}$ ratio (O/P ratio) for each sample, according to the equation derived from the linear regression analysis of the correlation between RTL and age (years) of the controls. We found that the mean O/P ratio of the patients $(0.83(0.20))$ was out of the $95 \%$ confidence limits of the controls (mean O/P ratio $0.67(0.17)$, $p=0.0226$ ), suggesting inappropriate MSC telomeric loss by age. By analysing the mean RTL per decade of years in patients and controls, a highly significant difference was obtained $(\mathrm{p}<0.001)$ (fig $3 \mathrm{~F})$, indicating further premature telomeric loss of MSCs in RA that might account, at least in part, for the defective clonogenic and proliferative potential of cells in culture.

\section{Gene expression profiling of MSCs}

Genes encoding for cell surface molecules, cytokines and differentiation markers were analysed in particular. Selection for genes differentially expressed in RA compared to normal MSCs allowed separation of the RA from normal samples by hierarchical clustering (fig 5). Affymetrix probe sets that were differentially expressed in more than $80 \%$ of the pairwise comparisons between three RA and six normal MSC samples were selected to identify objects in canonical pathways of the Kyoto Encyclopaedia of Genes and Genomes (KEGG) database. The most differentially expressed genes were those encoding for proteins implicated in focal adhesions (supplementary fig 1 and supplementary table 2). Specifically, several genes involved in cytoskeleton regulation and therefore linked to cell motility, proliferation and survival, were found to be differentially expressed between patients and controls. Of particular interest was the downregulation of cyclin-D, which plays an important role in the transition from G1 to S-phase in cell cycle progression, as well as the upregulation of cyclin-D inhibitors Ink4a-d and Kip1,2 and their upstream inductors $\mathrm{Smad} 2 / 3$ and TGF- $\beta$ (supplementary table 3). 

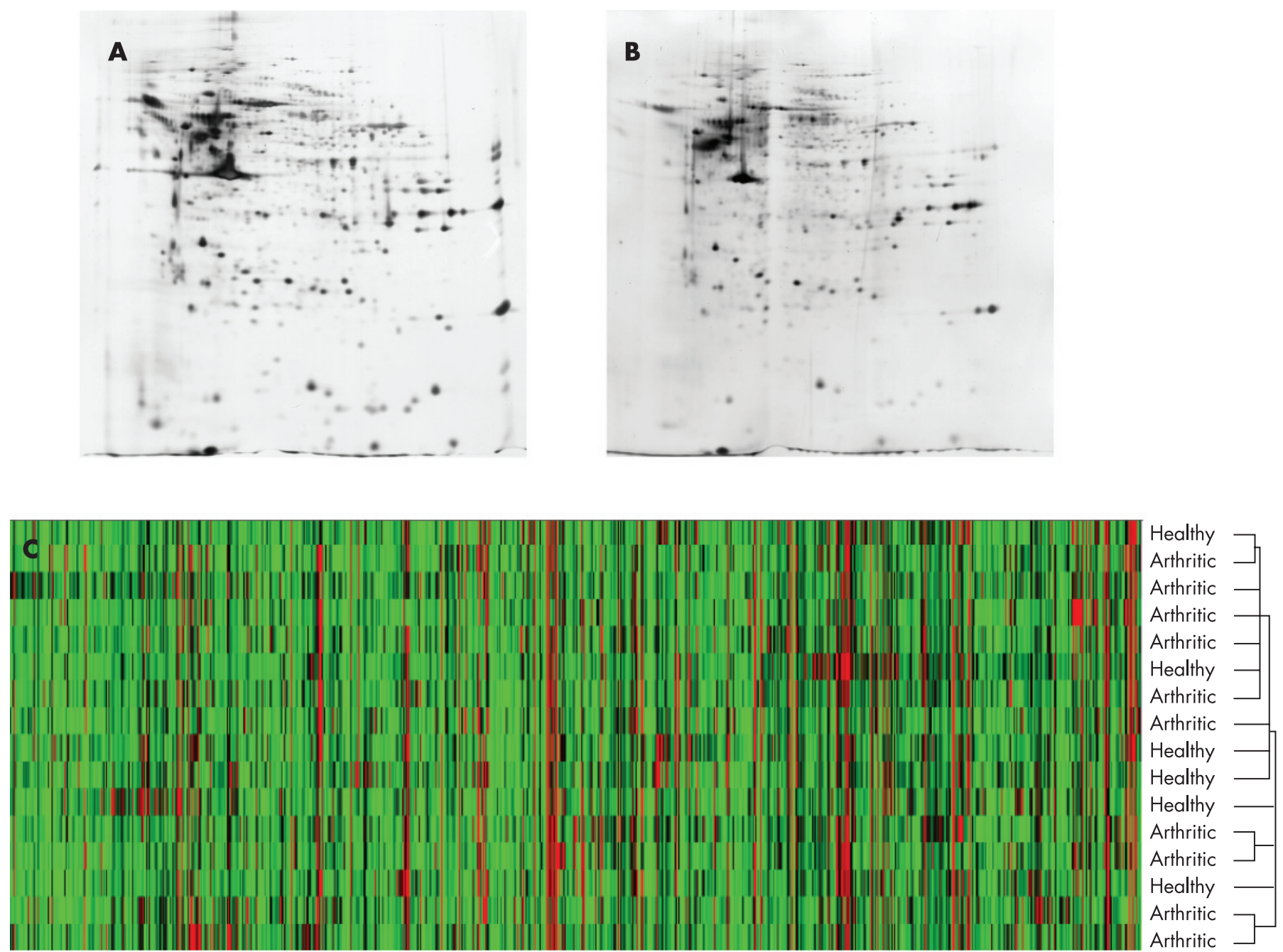

Figure 4 Proteomic analysis of mesenchymal stem cells (MSCs). Two-dimensional gel electrophoresis was performed using whole protein cell extracts from P2 MSC cultures of patients with rheumatoid arthritis $(R A)(n=10)(A)$ and healthy controls $(n=6)(B)$. After scanning, spot detection, quantification and normalisation, gels were compared using Hierarchical Clustering Software and Pearson test (C). No cluster could be detected using these proteomic profiles.

\section{DISCUSSION}

To explore the potential use of MSCs for cartilage and bone repair, we conducted the current study to evaluate the reserves, functional, molecular, proteomic characteristics, and differentiation potential of BM MSCs in patients with RA, a candidate disease characterised by destruction of articular structures. Characterisation of autologous MSCs in patients with RA is important since emerging data suggest that allogeneic MSCs may not be as "immune privileged" as previously considered. ${ }^{29}$

We found that the frequency of MSCs within BMMCs did not differ between patients and controls. Our findings are in accordance with previously reported data on the frequency of MSCs in normal BM suggesting that patients with RA have normal BM MSC reserves. ${ }^{40}$ Furthermore, culture-expanded MSCs from patients with RA were morphologically and immunophenotypically indistinguishable from the controls.

Culture-expanded MSCs from our patients with RA displayed normal osteogenic, chondrogenic and adipogenic differentiation. Interestingly, reduced chondrogenic activity of BM MSCs has been reported for osteoarthritis patients and this defect has been associated with the disturbed homeostatic maintenance of the articular cartilage. ${ }^{31}$ Normal differentiation potential of BM MSCs however, has been described in other autoimmune diseases such as in systemic sclerosis. ${ }^{32}$

MSC expansion through passages, assessed by the population doubling time, was significantly reduced in patients with RA, suggesting defective proliferative capacity. This finding was further substantiated by the MTT assay. The defective proliferative potential was in accordance with the impaired clonogenic capacity of patient MSCs as demonstrated by the low CFU-F recovery timecourse. In addition, the impaired growth of MSCs in RA is not likely to be due to accelerated cell apoptosis, since apoptosis in culture was not found to be significantly different between patients and controls.

The majority of our patients had been treated with methotrexate and/or corticosteroids, anti-cytokine or diseasemodifying anti-inflammatory agents. It has been reported that methotrexate does not affect the survival and functional characteristics of MSCs, while corticosteroids may damage their proliferative potential. ${ }^{33}$ No available data on the effect of anti-cytokine and anti-inflammatory agents on MSCs are presently known. To exclude the possibility of drug-mediated damage, we have separately analysed MSC clonogenic/proliferative potential in untreated patients. Cellular growth and 


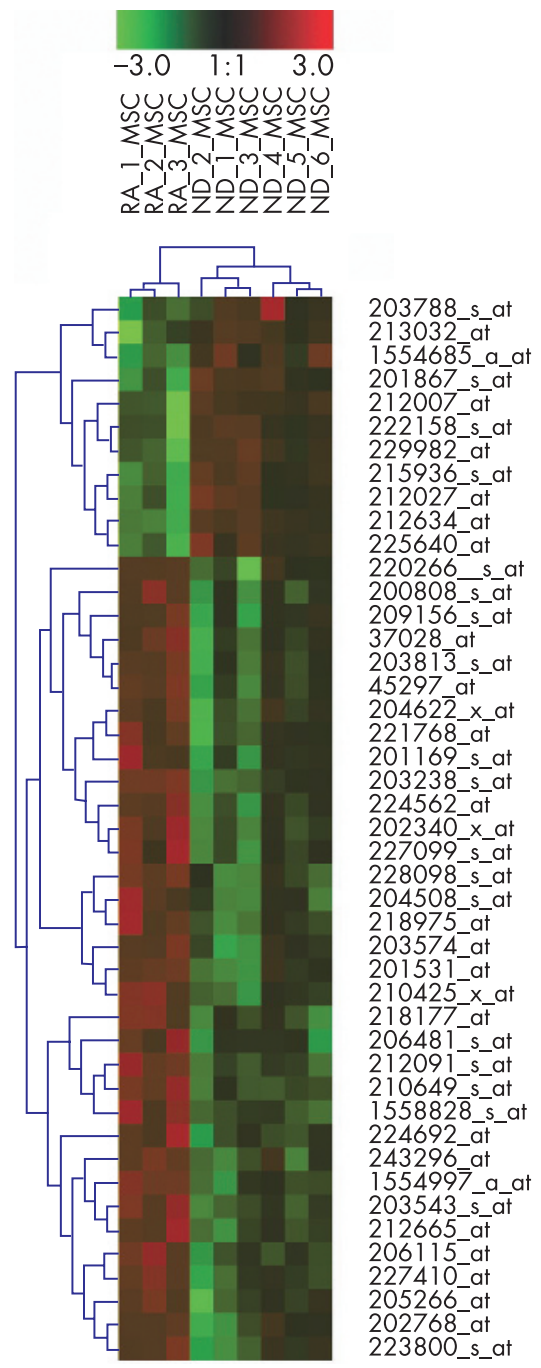

Sema domain. Immunoglobulin domain (lg). Short basic domain. Secreted. (Semaphorin) 3C Nuclear factor L/B

KIAA1199

Transducin (beta)-like 1X-linked

UBX domain containing 2

Chromosome 1 open reading frame 121

Glutamine and serine rich 1

KIAA1033

RNA binding motif protein 25

KIAA0776

Hypothetical gene supported by AK091718

Kruppel-like factor 4 (gut)

Zyxin

Collagen. Type VI. $\alpha 2$

Protein phosphatase 1. Regulatory (inhibitor) subunit 15A

Slit homolog 3 (Drosophila)

EH-domain containing 2

Nuclear receptor subtamily 4. Group A. Member 2

Splicing factor proline/glutamine-rich (polypyrimidine tract binding protein associated)

Basic helix-loop-helix domain containing. Class B. 2

Notch homolog 3 (Drosophila)

WAS protein famliy. Member 2

Nuclear receptor subfamily 4 . Group A. Member 1

Hypothetical LOC 387763

Myosin regulatory light chain interacting protein

Carbonic Anhydrase XI

Collagen. Type V. $\alpha 3$

Nuclear factor. Interleukin 3 regulated

Zinc finger protein 36 . C $3 \mathrm{H}$ type. Homolog (mouse)

Golgi autoantigen. Golgin subfamily a.8B

Chromatin modifying protein $1 \mathrm{~B}$

LIM domain binding 2

Collagen. Type VI. al

AT rich interactive domain 1A (SWI-like)

AT rich interactive domain $1 \mathrm{~A}$ (SWI-like
Hypothetical protein DKFZp586C0721

Protein phosphatase 1. Regulatory (inhibitor) subunit 15B

Pre-B-cell colony enhancing factor 1

Prostaglandin-endoperoxide synthase 2 (prostaglandin $\mathrm{G} / \mathrm{H}$ synthase and cyclooxygenase)

Kruppel-like factor 9

TCDD-inducible poly (ADP-ribose) polymerase

Early growth response 3

Family with sequence similarity 43. Member A

Leukemia inhibitory factor (cholinergic differentiation factor)

FBJ murine osteosarcoma viral oncogene homolog B

LIM and senescent cell antigen-like domains $3 / \% /$ similar to LIM and senescent cell antigen-like domains 3

Figure 5 Gene expression profiling of mesenchymal stem cells (MSCs). RNA from P2 bone marrow (BM) MSCs from patients with rheumatoid arthritis (RA) $(n=3)$ and healthy controls $(n=6)$ was hybridised to the GeneChips and arrays were processed for signal calculation and pairwise chip comparison. Selection of genes differentially expressed in RA compared to non-differentiated (ND) bone marrow mononuclear cells (BMMCs) allowed us to distinguish RA MSCs from normal MSCs by hierarchical clustering.

CFU-F recovery were significantly lower even in this group of patients compared to controls, whereas no significant differences were documented between treated and untreated patients with RA. These findings suggest that treatment with antirheumatic agents is not the major factor affecting MSC growth in RA.

It has been suggested that BM derived MSCs with altered properties may repopulate the synovial membrane in RA, whereas abnormal expression of inflammatory cytokines has been reported for RA synovial fibroblasts. ${ }^{12}{ }^{13}$ MSCs from our patients displayed normal cytokine expression in array profiling and ELISA. We have specifically focused on IL1 $\beta$ and TNF $\alpha$ since these cytokines have been found overexpressed in synovial fluid and BM culture supernatants. ${ }^{14}{ }^{34}{ }^{35}$ No differences were found between patients and controls in the inflammatory cytokine expression or the growth promoting cytokine levels, suggesting that the impaired growth potential found in patient MSCs is not due to altered cytokine expression.

It has been previously shown that aging may affect the proliferative potential of MSCs. ${ }^{36}$ Since patients with RA and controls in our study were age-matched, an age-related defect in the clonogenic and proliferative capacity of patient MSCs is unlikely. Patient MSCs, however, displayed age-inappropriate relative telomere loss. Since there is evidence suggesting that the replicative capacity of cells correlates with the initial cellular telomere length ${ }^{37}{ }^{38}$ we may speculate that the defective growth potential of patient MSCs is due to inappropriate telomere loss. Although telomerase activity was not assessed in our study, we hypothesise that it is under the influence of the inflammatory BM milieu. ${ }^{14}$ MSCs in RA undergo accelerated proliferation resulting in premature replicative exhaustion as previously described for RA periarticular osteoblasts. ${ }^{28}$ The recently described accelerated telomere shortening under the influence of chronic mild oxidative stress further corroborates this assumption. ${ }^{24}$

In the search for direct molecular mechanisms by array profiling, differentially expressed genes were found to be involved especially in cell adhesion processes and matrix (collagen) synthesis. In particular, downregulation of cyclin-D was identified. Thus, a repressive influence on the transition from G1 to S-phase could provide a direct molecular explanation for the altered proliferative capacity of MSCs from patients with RA. This could be related to the increased local production of TGF- $\beta$ in patients' BM microenvironment (data not shown) 
that may result in increase of TGF- $\beta$ signalling in MSCs and consecutive expression of cyclin-dependent kinase inhibitors, as suggested from the "cell cycle" pathway from KEGG. Thus, MSCs from patients with RA may present in vitro with a phenotype of cells that are more adherent, produce more matrix and are less active in proliferation. However, functional data are needed to substantiate this hypothesis.

In conclusion, our data have shown that patients with RA display a normal number, molecular and proteomic profile and differentiation potential of BM MSCs, encouraging, therefore, the use of autologous MSCs for the repair of cartilage and bone damage associated with long-standing RA. The proliferative and clonogenic potential of patient BM MSCs, however, was reduced compared to normal MSCs, and this defect was associated with decreased cellular telomere length and altered expression of genes implicated in focal adhesion and cell cycle pathways. This altered MSC activity may potentially influence the preparation of the cells for therapeutic usage in RA. In summary, this is the first study characterising BM MSCs in patients with RA under defined culture conditions in the context of potential clinical use for the repair of tissue injury associated with RA.

Acknowledgements: The authors wish to thank Mrs Helen Koutala, Mrs Claudia Gemetzi and Mrs Athina Damianaki (University of Crete, Greece) for their valuable technical assistance.

Funding: This study was supported by the 6th European Union Framework Program (FP6, GENOSTEM \#503161).

Competing interests: None declared.

Ethics approval: The Institutional ethics committee approved the study and informed consent according to the Helsinki Declaration was obtained from all subjects.

\section{REFERENCES}

1. Owen M. Marrow stromal cells. J Cell Sci Supp/ 1985;10:63-76.

2. Bianco $\mathbf{P}$, Riminucci $M$, Gronthos $S$, Robey PG. Bone marrow stromal stem cells: nature, biology, and potential applications. Stem Cells 2001;19:180-92

3. Gronthos S, Simmons PJ. The biology and application of human bone marrow stromal cell precursors. J Hematother 1996;5:15-23.

4. Pittenger MF, Mackay AM, Beck SC, Jaiswal RK, Douglas R, Mosca JD, et al. Multilineage potential of adult human mesenchymal stem cells. Science 1999:284:143-7.

5. Deans RJ, Moseley AB. Mesenchymal stem cells: biology and potential clinical uses. Exp Hematol 2000;28:875-84.

6. Barry FP, Murphy JM. Mesenchymal stem cells: clinical applications and biological characterization. Int J Biochem Cell Biol 2004;36:568-84.

7. Otto WR, Rao J. Tomorrow's skeleton staff: mesenchymal stem cells and the repair of bone and cartilage. Cell Prolif 2004;37:97-110.

8. Jorgensen C, Gordeladze J, Noel D. Tissue engineering through autologous mesenchymal stem cells. Curr Opin Biotechnol 2004:15:406-10.

9. Mankani MH, Kuznetsov SA, Fowler B, Kingman A, Robey PG. In vivo bone formation by human bone marrow stromal cells: effect of carrier particle size and shape. Biotechnol Bioeng 2001:72:96-107.

10. Tsuchida H, Hashimoto J, Crawford E, Manske P, Lou J. Engineered allogeneic mesenchymal stem cells repair femoral segmental defect in rats. J Orthop Res 2003;21:44-53.

11. Nakagawa S, Toritsuka Y, Wakitani S, Denno K, Tomita T, Owaki H, et al. Bone marrow stromal cells contribute to synovial cell proliferation in rats with collagen induced arthritis. J Rheumatol 1996;23:2098-103.

12. Suzuki Y, Nishikaku F, Nakatuka M, Koga Y. Osteoclast-like cells in murine collagen induced arthritis. J Rheumatol 1998:25:1154-60.

13. Sen M, Lauterbach K, El-Gabalawy H, Firestein GS, Corr M, Carson DA. Expression and function of wingless and frizzled homologs in rheumatoid arthritis. Proc Natl Acad Sci USA 2000:97:2791-6.

14. Papadaki HA, Kritikos HD, Gemetzi C, Koutala H, Marsh JC, Boumpas DT, et al. Bone marrow progenitor cell reserve and function and stromal cell function are defective in rheumatoid arthritis: evidence for a tumor necrosis factor alpha-mediated effect. Blood 2002;99:1610-19.
15. Arnett FC, Edworthy SM, Bloch DA, McShane DJ, Fries JF, Cooper NS, et al. The American Rheumatism Association 1987 revised criteria for the classification of rheumatoid arthritis. Arthritis Rheum 1988;31:315-24.

16. Pincus T, Sokka T. Quantitative measures for assessing rheumatoid arthritis in clinical trials and clinical care. Best Pract Res Clin Rheumatol 2003;17:753-81.

17. In't Anker PS, Noort WA, Scherjon SA, Kleijburg-van der KC, Kruisselbrink AB, van Bezooijen RL, et al. Mesenchymal stem cells in human second-trimester bone marrow, liver, lung, and spleen exhibit a similar immunophenotype but a heterogeneous multilineage differentiation potential. Haematologica 2003:88:845-52.

18. Delorme B, Charbord P. Culture and characterisation of human bone marrow mesenchymal stem cells. In: Hauser $\mathrm{H}$, Fussenegger $\mathrm{M}$, eds. Methods in molecular medicine, 2nd edn: tissue engineering. Totowa, New Jersey, USA: Humana Press Inc, 2007.

19. Gronthos S, Graves SE, Simmons PJ. Isolation, purification and in vitro manipulation of human bone marrow stromal precursor cells. In: Beresford JN, Owen ME, eds. Marrow stromal cell culture. Cambridge, UK: Cambridge University Press, 1998:26-42.

20. Lisignoli G, Cristino S, Piacentini A, Zini N, Noel D, Jorgensen C, et al. Chondrogenic differentiation of murine and human mesenchymal stromal cells in a hyaluronic acid scaffold: differences in gene expression and cell morphology. J Biomed Mater Res A 2006; 77:497-506

21. Philpott NJ, Turner AJ, Scopes J, Westby M, Marsh JC, Gordon-Smith EC, et al. The use of 7-amino actinomycin D in identifying apoptosis: simplicity of use and broad spectrum of application compared with other techniques. Blood 1996;87:2244-51.

22. Rizzo S, Scopes J, Elebute MO, Papadaki HA, Gordon-Smith EC, Gibson FM. Stem cell defect in aplastic anemia: reduced long term culture-initiating cells (LTC-IC) in CD34+ cells isolated from aplastic anemia patient bone marrow. Hematol $\mathrm{J}$ 2002;3:230-6.

23. Cawthon RM. Telomere measurement by quantitative PCR. Nucleic Acids Res 2002;30:e47

24. Martin-Ruiz C, Saretzki G, Petrie J, Ladhoff J, Jeyapalan J, Wei W, et al. Stochastic variation in telomere shortening rate causes heterogeneity of human fibroblast replicative life span. J Biol Chem 2004;279:17826-33.

25. Shevchenko A, Wilm M, Vorm 0, Mann M. Mass spectrometric sequencing of proteins silver-stained polyacrylamide gels. Anal Chem 1996;68:850-8.

26. Seo J, Bakay M, Chen YW, Hilmer S, Shneiderman B, Hoffman EP. Interactively optimizing signal-to-noise ratios in expression profiling: project-specific algorithm selection and detection p-value weighting in Affymetrix microarrays. Bioinformatics 2004:20:2534-44.

27. Takeuchi E, Tomita T, Toyosaki-Maeda T, Kaneko M, Takano H, Hashimoto H, et al Establisment and characterization of nurse-like stromal cell lines from synovial tissues of patients with rheumatoid arthritis. Arthritis Rheum 1999:42:221-8.

28. Yudoh K, Matsuno H, Osada R, Nakazawa F, Katayama R, Kimura T. Decreased cellular activity and replicative capacity of osteoblastic cells isolated from the periarticular bone of rheumatoid arthritis patients compared with osteoarthritis patients. Arthritis Rheum 2000;43:2178-88.

29. Nauta AJ, Westerhuis G, Kruisselbrink AB, Lurvink EG, Willemze R, Fibbe WE. Donorderived mesenchymal stem cells are immunogenic in an allogeneic host and stimulate donor graft rejection in a nonmyeloablative setting. Blood 2006:108:2114-20.

30. Caplan Al. The mesengenic process. Clin Plast Surg 1994;21:429-35.

31. Murphy JM, Dixon K, Beck S, Fabian D, Feldman A, Barry F. Reduced chondrogenic and adipogenic activity of mesenchymal stem cells from patients with advanced osteoarthritis. Arthritis Rheum 2002;46:704-13

32. Larghero J, Farge D, Braccini A, Lecourt S, Scherberich A, Fois E, et al. Phenotypical and functional characteristics of in vitro expanded bone marrow mesenchymal stem cells from systemic sclerosis patients. Ann Rheum Dis 2008;67:443-9.

33. Li J, Law HK, Lau YL, Chan GC. Differential damage and recovery of human mesenchymal stem cells after exposure to chemotherapeutic agents. $\mathrm{Br} J$ Haemato 2004;127:326-34.

34. Papadaki HA, Kritikos HD, Valatas V, Boumpas DT, Eliopoulos GD. Anemia of chronic disease in rheumatoid arthritis is associated with increased apoptosis of bone marrow erythroid cells: improvement following anti-tumor necrosis factor-alpha antibody therapy. Blood 2002;100:474-82

35. Lisignoli G, Toneguzzi S, Pozzi C, Piacentini A, Riccio M, Ferruzzi A, et al Proinflammatory cytokines and chemokine production and expression by human osteoblasts isolated from patients with rheumatoid arthritis and osteoarthritis. J Rheumatol 1999;26:791-9

36. Mareschi K, Ferrero I, Rustichelli D, Aschero S, Gammaitoni L, Aglietta M, et al. Expansion of mesenchymal stem cells isolated from pediatric and adult donor bone marrow. J Cell Biochem 2006;97:744-54.

37. Harley CB, Futcher $A B$, Greider CW. Telomeres shorten during ageing of human fibroblasts. Nature 1990;345:458-60.

38. Levy MZ, Allsopp RC, Futcher AB, Greider CW, Harley CB. Telomere end-replication problem and cell aging. J Mol Biol 1992;225:951-60. 